\title{
Automated counting of bacterial colonies by image analysis
}

\section{Shashidara Raju ${ }^{1}$, Aparna H G ${ }^{2}$, Archana Vaikom Krishnan ${ }^{3}$, Dhanesh Naryanan ${ }^{4}$, Vishwajith Gangadhran ${ }^{5}$, Shelma C Paul ${ }^{6}$}

1. Dr. Shashidara Raju, Professor \& Head of the Department of Oral Pathology, Coorg Institute of Dental Sciences

2. Dr. Aparna H G- Professor, Coorg Institute of Dental Sciences

3. Dr. Archana Vaikom Krishnan- Senior Lecturer, Coorg Institute of Dental Sciences

4. Dr. Dhanesh Naryanan- Senior Lecturer, Coorg Institute of Dental Sciences

5. Mr Viswajith Gangadharan- Undergraduate, Coorg Institute of Dental Sciences

6. Ms Shelma C Paul- Undergraduate, Coorg Institute of Dental Sciences

Corresponding author:

Dr. Shashidara Raju

Professor \& Head of the Department of Oral Pathology

Coorg Institute of Dental Sciences,

Maggula post

Virajpet-571218

\begin{abstract}
Colony counting is some of the most basic and frequent operation in colony cultivating trials, and is both basic and important in agriculture, foods, medical analysis. Currently, people adopt national standard detection technique to count colonies. When the sample is huge, the method becomes difficult and time consuming. It is necessary to enhance the traditional counting method with the advancement of computer technology. We therefore implemented a colony counting system with a Biowizard Image Analysis Software to count the bacteria in the agar plates. The automatic colony counting program was user friendly and did not require any specific hardware or extensive training. It only required a digital image of the agar plates with colonies in any image format. The Biowizard Image Analysis Software gave better information about colony parameters, with respect to sensitivity and time required, as compared to manual counting and other commercial softwares.
\end{abstract}

Keywords: Colony counting, Microbiology, Biowizard, Analysis Software 


\section{Introduction}

Microbiological study methods are often depending on the exact determination of colony forming units (CFUs). Regularly, this is prepared by aliquoting a small amount of a liquid culture and plating out some serial dilutions onto the culture plates. Once the incubation is done for suitable conditions of the microorganism of choice, the colonies are counted to decide the amount of CFU. This is done by calculating the number of colonies on plates illuminated by transmitted light. ${ }^{1}$ Counting cell colonies are essential for assessing microbial content, assessing cytotoxicity and the function of particular genes in microbiology, immunology, and cell biology. ${ }^{2}$

Colony counting is some of the most basic and frequent operations in colony cultivating trials and is both basic and important in agriculture, foods, medical analysis. Currently, people adopt the national standard detection technique for calculating colony, which is the conventional nutrition agar pump way. Once the quantity of sample is huge, the technique becomes difficult, time-consuming, low efficiency, so it is required to enhance the traditional counting method with the advancement of computer technology, by using computer device to effectively reduce the intensity of production, improve labor productivity, and realize production automation has become a development trend. In recent years, with the help of computer processing technology to solve the quality detection task of agricultural products has been the overall concern of scholars at home and abroad, so as to colony counting.

The content of bacteria in the original culture can then be calculated based on the assumption that each colony has raised from one single bacterium colony forming unit (CFU). This process is timeconsuming, tedious and prone to error. Manual calculation of the bacterial colony forming units (CFUs) on agar plates is difficult and error-prone.
We, therefore, implemented a colony counting system with a Biowizard Image Analysis Software to count the bacterial in the agar plates ${ }^{1}$.

\section{Materials And Methods}

Quantitative assessment of the live microbial cell concentration/content in various samples by their growth in Petri dishes up to the visible colonies is one of the most common assays in microbiology. Though, visual/manual colony counting is very tedious and subjective. Hence, interest in automating the colony counting procedure arose long ago. The first device for automated colony counting was described in 1957. It worked by scanning the Petri dish image on a CRT screen by a photomultiplier and processing of the digitized image by a computer. ${ }^{4}$

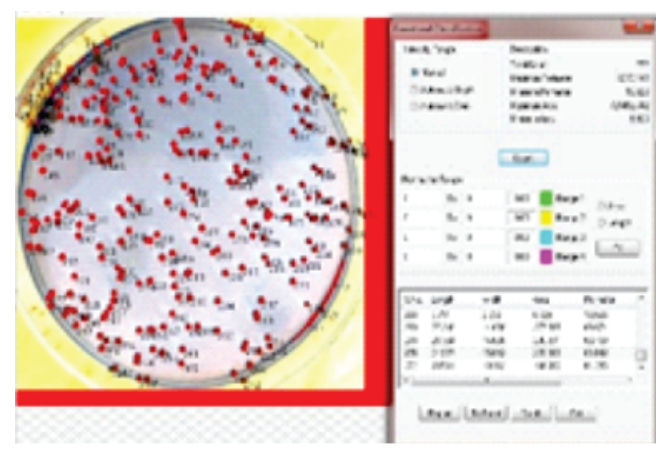

FIG.1 - ANALYSIS SOFWARE

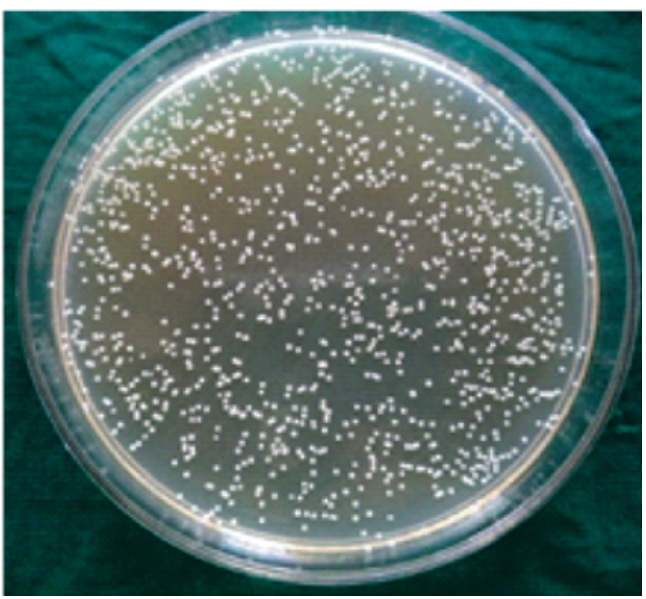

FIG. 2 - PLATE WITH CANDIDAL GROWTH 
The principal parts of this type of colony counters are a camera to capture digital images of the Petri dishes and a computer with an appropriate software. We have used Biowizard Image Analysis Software to do automatic colony counting. (Figure 1) We took one plate with the candidal growth and captured an image using Sony cyber shot (N 50) (Figure 2) and took the round areas of interest. The images were then converted to black and white and inverted the images. Post inverting of the image contrast enhancement was done and the colonies was counted using the manual intensity and adjustment.

\section{Results}

The results obtained using this program were compared with the manual counting as well as with automated counting Biowizard Image Analysis Software. It was found that our results was that to be good enough when comparing with manual counting.

\section{Discussion \& Conclusion}

Due to extensive use of clonogenic assay in the field of toxicology, cancer biology and radiation biology, a user-friendly and automated and semiautomated colony counting program is required for fast data analysis over manual counting. According to a study done by Mayura at el in 2017 found that both GelCount ${ }^{\mathrm{TM}}$ and ColonyCount $\mathrm{yielded}$ comparable outcomes in terms of the number of colonies. ${ }^{5} \quad$ Chiang et al in 2015 showed that performance of the proposed system is compared with verified manual counts, as well as with two freely available counting software programs. Comparisons show that the proposed system is an effective method with excellent accuracy with a mean value of absolute percentage error of $3.37 \%{ }^{6}$
In conclusion, the automatic colony counting program, Biowizard Image Analysis Software here is user-friendly and does not require any specific hardware and extensive training on the software. It requires a digital image of the colonies in any image format. Biowizard Image Analysis Software gave better information about colony parameters (sensitivity and time required) as compared to manual and other commercial software.

\section{References}

1) Brugger $\mathrm{SD}$, Baumberger $\mathrm{C}$, Jost $\mathrm{M}$, Jenni $\mathrm{W}$, Brugger U, Mühlemann K. Automated counting of bacterial colony forming units on agar plates. PloS one. 2012 Mar 20; 7(3):e33695.

2) Choudhry P. High-throughput method for automated colony and cell counting by digital image analysis based on edge detection. PLoS One. 2016 Feb 5;11(2):e0148469.

3) Fang J, Li W, Wang G. Experimental Study for Automatic Colony Counting System Based Onimage Processing. InInternational Conference on Computer and Computing Technologies in Agriculture 2008 Oct 18 (pp. 1061-1066). Springer, Boston, MA.

4) Puchkov E. Image analysis in microbiology: A review. J. Comput. Commun. 2016 Nov 15;4(15):72308.

5) Maurya DK (2017) ColonyCountJ: A UserFriendly Image J Add-on Program for Quantification of Different Colony Parameters in Clonogenic Assay. J ClinToxicol 7: 358.

6) Chiang PJ, Tseng MJ, He ZS, Li CH. Automated counting of bacterial colonies by image analysis. Journal of microbiological methods. 2015 Jan 1; 108: 74-82. 